\title{
Analysis of Vertical Stiffness of Air Spring Based on Finite Element Method
}

\author{
Jiatong Ye, Hua Huang, Chenchen He, Guangyuan Liu \\ School of mechanical and Vehicular Engineering, Beijing Institute of Technology, 100081 China
}

\begin{abstract}
In this paper, a finite element model of membrane air spring in the vehicle is established, and its vertical stiffness characteristics under a certain inflation pressure are analysed. The result of finite element simulation method is compared with the result of the air spring bench test. The accuracy and reliability of the finite element simulation method in nonlinear analysis of air spring system are verified. In addition, according to the finite element method, the influence of the installation of the air spring limit sleeve on its stiffness is verified.
\end{abstract}

\section{Introduction}

As the air spring has great advantages in the vibration performance and energy storage and other aspects, relative to other elastic components, the air spring gains more and more popularity in consumers and developers of all ages. The stiffness of the air spring is an important performance parameter involved in the design and application of air springs. Analysing the stiffness of the air spring with finite element method can take the impact of each parameter on the stiffness into account synthetically, greatly shortening the development cycle[1]. Therefore, the finite element method has great theoretical and practical value for the research and analysis of air springs. Based on the advantages above, the stiffness of a certain type of spring is analysed by finite element method, and the correctness of the analysis is proved by comparison with the experimental results.

\section{Vertical stiffness of air spring}

The force diagram of air spring is shown in figure 1 .

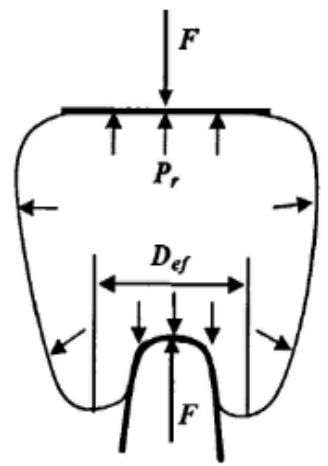

Figure 1.The force diagram of air spring.
The relationship between the vertical load $F$, the relative internal pressure $P_{r}$, the effective bearing area $A_{e f}$ and the effective diameter $D_{e f}$ on the air spring is:

$$
F=\mathrm{P}_{r} A_{e f}=\frac{\pi}{4} \mathrm{P}_{r} D_{e f}^{2}
$$

If the quality of compressed gas does not change, the load change will cause the height change of the air spring, which will lead to changes in the volume and pressure of the airbag. the process of change meets the ideal gas equation:

$$
\left(\mathrm{P}_{r}+\mathrm{P}_{a}\right) V^{n}=\left(\mathrm{P}_{0}+\mathrm{P}_{a}\right) V_{0}^{n}
$$

Where: $P_{a}$ is the atmospheric pressure, $P_{0}$ is the initial internal pressure of the air spring, $V_{0}$ is the initial volume of the air spring; $V$ is the volume of the airbag at any position, $P_{r}$ is the internal pressure in the airbag at any position; $n$ is the polytropic index, in the range of 1 to 1.4 .

Sort out the formula above, its vertical stiffness can be obtained by deriving the stroke $x$ :

$$
k=\frac{d F}{d x}=-n A_{e f} \frac{\mathrm{P}_{r}+\mathrm{P}_{a}}{V} \frac{d V}{d x}+\mathrm{P}_{r} \frac{d A_{e f}}{d x}
$$

Where $\frac{d V}{d x}$ is the volume change rate of the air spring and $A$ is the effective area change rate of the air spring[2].

\section{Finite element analysis of membrane air spring}

\subsection{The establishment of finite element model for membrane air spring}


In this study, the effects of the emergency stop, stud and bolt holes are ignored because the emergency stop only operate under extreme conditions, and the stud and bolt holes were independent of the product performance. As a result, the key point of finite element analysis is focused on the shape of the piston, the capsule and the edge of the lid. Experience shows that the piston and the lid should be seen as rigid body structure, because the upper and lower bead ring fixed in the lid edge and the piston, so it can be ignored in finite element analysis[3].

The airbag of the membrane air spring is made of a composite material obtained by vulcanization of rubber and cord. Because of the limit deformation degree of the rubber of the membrane air spring airbag, the rubber has the same nature in the small deformation period, and the order of the macromolecular chains has not been affected. The superelastic mechanical properties can be described by Mooney-Rivlin model[4] strain energy density function $U$, that is

$$
U=C_{10}\left(I_{1}-3\right)+C_{01}\left(I_{2}-3\right)+\frac{1}{D}\left(J^{e l}-1\right)^{2}
$$

Where $C_{10} 、 C_{01}$ and $D$ are temperature-dependent superelastic material constants, which can be obtained by testing the material; $J^{e l}$ is the elastic volume ratio; $I_{l}$ and $I_{2}$ are the first and second strain invariants, which are defined as:

$$
\begin{gathered}
I_{1}=\lambda_{1}^{2}+\lambda_{2}^{2}+\lambda_{3}^{2} \\
I_{2}=\lambda_{1}^{-2}+\lambda_{2}^{-2}+\lambda_{3}^{-2}
\end{gathered}
$$

Where $\lambda_{1} 、 \lambda_{2}$ and $\lambda_{3}$ are the tensile coefficients of the three main stretching directions.

The initial shear modulus and bulk modulus of elasticity are:

$$
\begin{gathered}
\mu_{0}=2\left(C_{10}+C_{01}\right) \\
K_{0}=\frac{D}{2}
\end{gathered}
$$

In this experiment, in order to control the deformation degree of the air spring balloon better, so as to obtain the air spring stiffness curve which is more in accordance with the actual situation, a more rigid limit sleeve made by aluminum alloy is set on the airbag surface. The discrete rigid body unit RAX2 can be used to simulate the limit sleeve. The piston and the upper cover are also simulated using the RAX2 discrete rigid body unit. The cord layer is a very important bearing component of the airbag. The ABAQUS software provides a rebar unit for simulating the stiffened ribbed structure. The rebar unit in the cord layer structure is usually embedded in the rubber shell model to form the airbag compound material[5]. The cord parameters of the air spring selection are shown in Table 1.

Table 1. Membrane air spring cord parameters

\begin{tabular}{|c|c|}
\hline Cord angle / $\left({ }^{\circ}\right)$ & \pm 34 \\
\hline Number of layers & 2 \\
\hline Spacing $/ \mathrm{mm}$ & 0.30 \\
\hline
\end{tabular}

\begin{tabular}{|c|c|}
\hline Diameter $/ \mathrm{mm}$ & 0.39 \\
\hline $\begin{array}{c}\text { Distance from the } \\
\text { neutral surface /mm }\end{array}$ & \pm 0.38 \\
\hline
\end{tabular}

There's no heat transfer to the outside when the air spring is compressed or stretched in a short time period ,so the process of change is similar to the adiabatic process. A hydrostatic fluid unit may be used: a model such as F3D3 (three-dimensional three-node fluid unit) or F3D4 (three-dimensional four-node fluid unit) as this fluid.

\subsection{Finite element analysis of air spring}

This paper uses ABAQUS for finite element analysis. Based on the piston restraint condition and the rigid freedom level of the upper cover, the corresponding initial pressure is determined in conjunction with the gas unit reference point data to meet the system working requirements and is adjusted to the working condition by inflating the rubber air bag. According to a similar method, the vertical displacement parameter of the upper cover plate is evaluated and the expected vertical movement distance of the air spring is determined, and the ideal height is determined. Then, with reference to the constraints of upper cover and the free movement of the piston, the ideal working pressure of the air spring is determined and inflate to ideal value[6]. Here, select the same conditions with the test, that is, inflate to the static balance height $\mathrm{H}=320 \mathrm{~mm}$, inflation pressure is $0.6 \mathrm{MPa}$. This inflated pressurization process is shown as figure 2 .

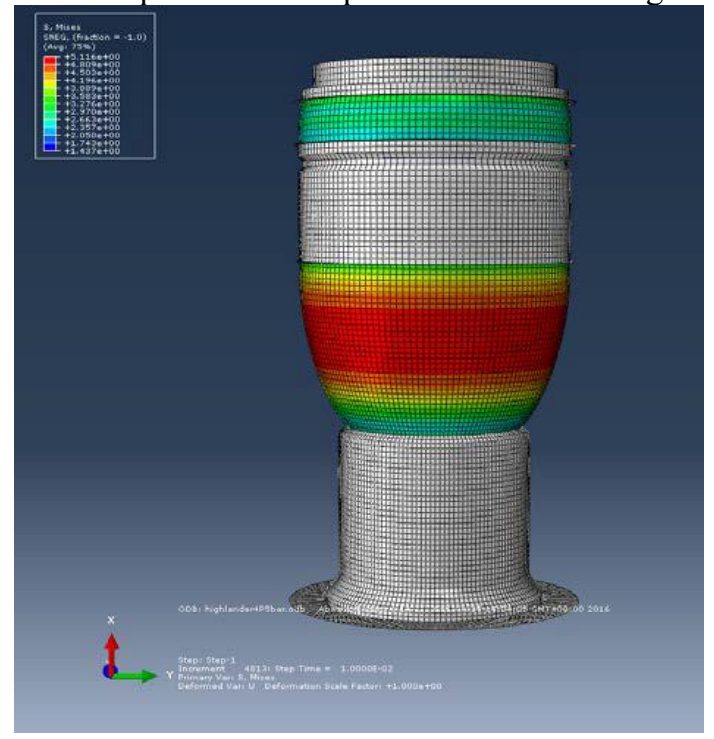

Figure 2.The stress cloud at the equilibrium position.

Based on the vertical travel of the upper cover, the load of air spring can be determined, and the confirmation method is similar to the analysis method of the rigid surface constraint condition of the upper cover. This paper analyzes the load fluctuation caused by stretching and compression with the change of displacement of the air spring, and then evaluates the vertical stiffness performance of the air spring. After the spring is inflated, 
the compressive stress and the tensile stress are shown in figure 3 .

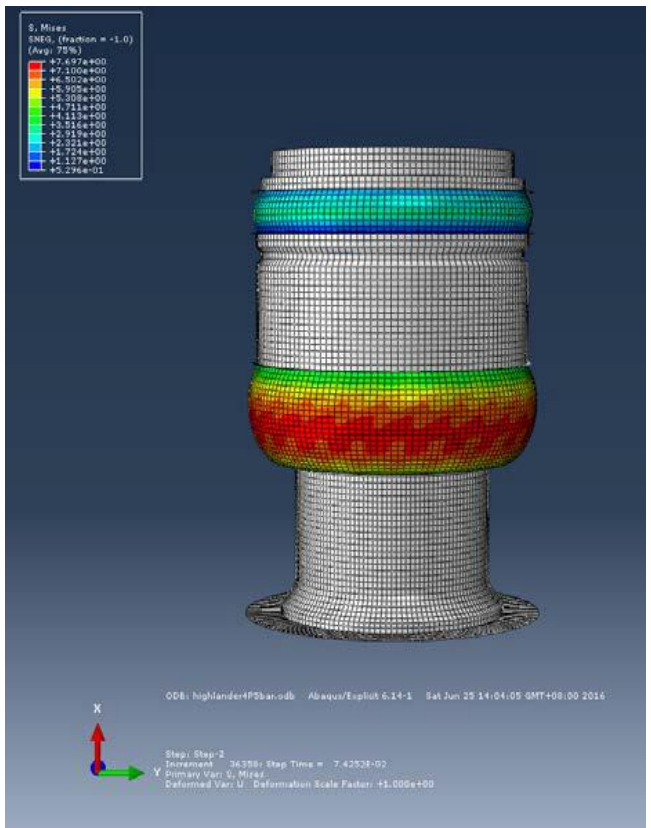

Figure 3.1. Compressive stress cloud.

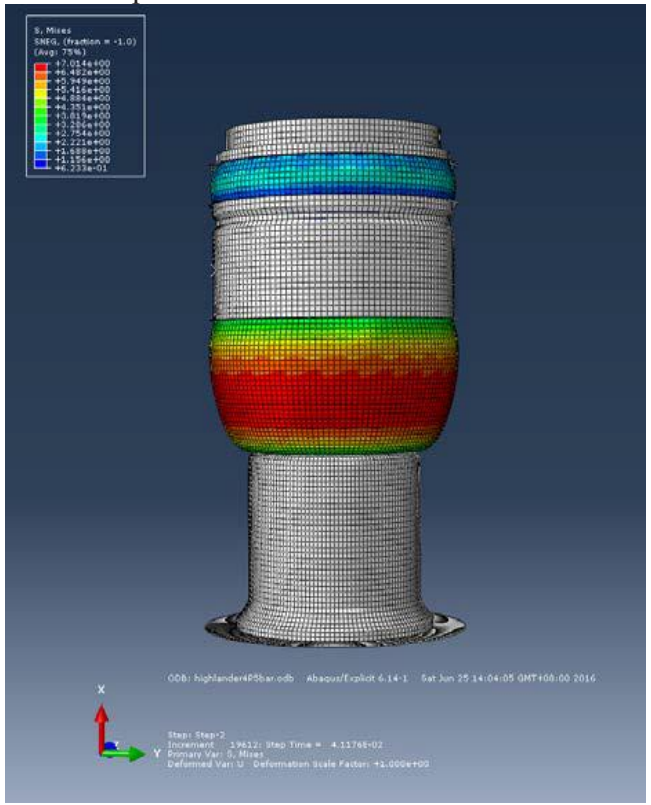

Figure 3.2.Tensile stress cloud.

\subsection{Influence of limit sleeve on vertical stiffness of air spring}

In order to verify the effect of the air spring limit sleeve on its stiffness, the limit sleeve is removed and the finite element calculation is performed again. To ensure that the two prerequisites for calculation such as the boundary conditions, displacement levels and other parameters are kept fixed. The results of the two finite element analysis are processed, and the force-displacement data are shown in figure 4.

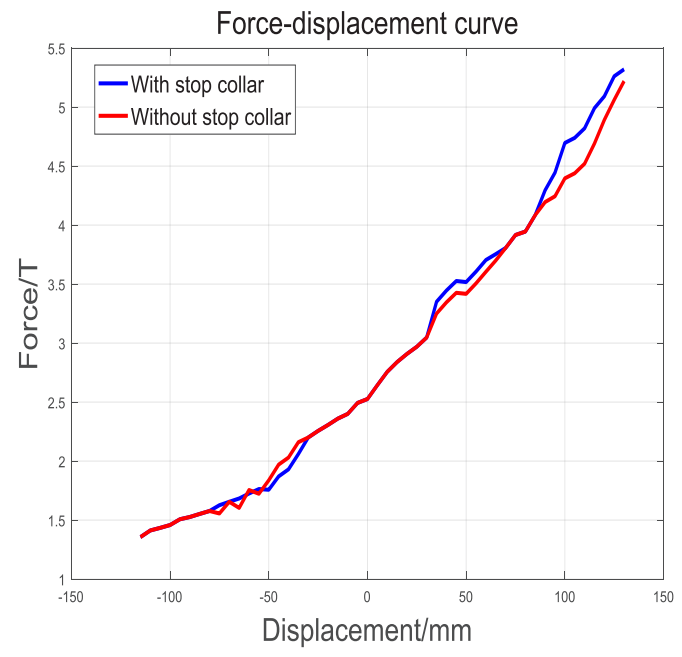

Figure 4.Force - displacement curve of air spring.

In order to facilitate the comparison with the subsequent test data, here take the data in the range of $50 \mathrm{~mm}$, draw the air spring static stiffness curve of the two finite element calculations, which are shown in figure 5.

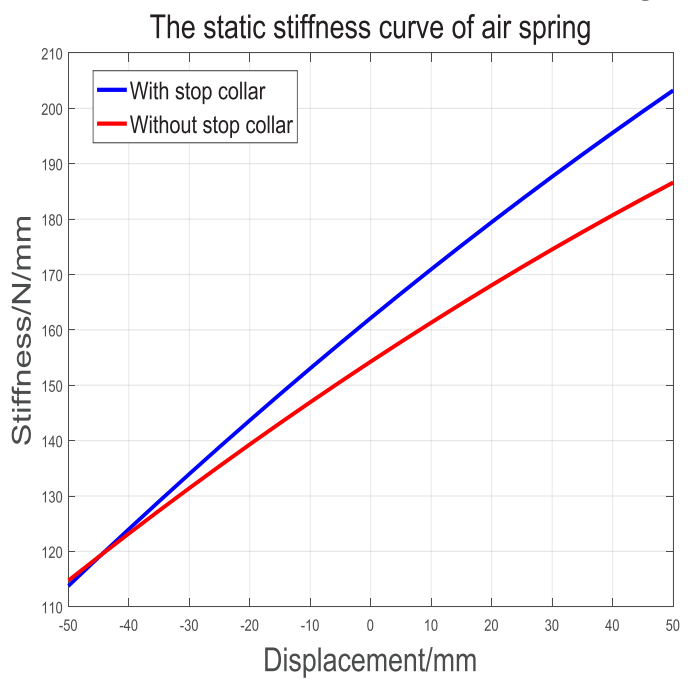

Figure 5.Comparison curve of static stiffness of air spring.

As can be shown from the curve comparison chart, the rigidity level of the air spring is improved after the installation of the limit sleeve, and its overall performance is improved synchronously. Besides, the excessive deformation of the airbag can be effectively suppressed to avoid the effect on the safety of the equipment.

\section{Vertical static characteristic test of air spring}

\subsection{Test of air spring}

The air spring is connected to the test bench through the flange connector and test bench gantry, as shown in figure 6 . 


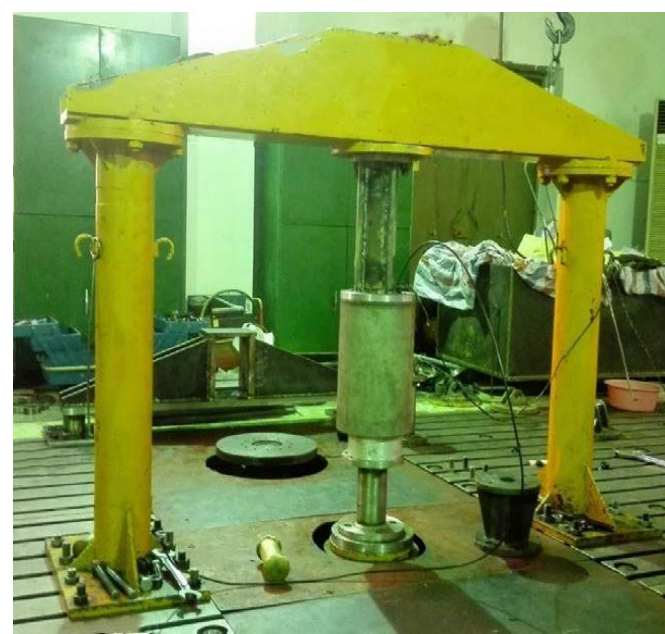

Figure 6.Test piece installation diagram. 2 .

The parameters of air spring assembly shown in Table

Table 2.Air spring assembly parameters

\begin{tabular}{|c|c|}
\hline Name & Parameters \\
\hline $\begin{array}{c}\text { Air spring assembly } \\
\text { maximum height }\end{array}$ & $470 \mathrm{~mm}$ \\
\hline $\begin{array}{c}\text { Air spring assembly } \\
\text { minimum height }\end{array}$ & $170 \mathrm{~mm}$ \\
\hline Static balance height & $320 \mathrm{~mm}$ \\
\hline Stroke & $\pm 150 \mathrm{~mm}$ \\
\hline Piston diameter & $180 \mathrm{~mm}$ \\
\hline
\end{tabular}

Adjust the air spring to the maximum height, inflate to 6bar. The specimen is run under $0.1 \mathrm{~Hz}$ sine excitation 10 times in the $80 \%$ of the stroke range. Adjust the $\mathrm{H}$ to $460 \mathrm{~mm}$, each time when the test bed rise $5 \mathrm{~mm}$, read the spring elastic force, until $\mathrm{H}$ reaches $180 \mathrm{~mm}$. And similarly drop the test bed every $5 \mathrm{~mm}$, read again until $\mathrm{H}$ reaches $460 \mathrm{~mm}$. In the compression and recovery process, due to airbag capsule friction and air through the orifice, the resulting damping forces are different. In addition, the existence of a variety of uncertain factors, the static force and pressure in test will also be different.

According to the original data obtained from the experiment, the static displacement - elastic curve in \pm $50 \mathrm{~mm}$ stroke is drawn and the corresponding fitting curve equation is obtained[7]. Based on the fitting equation, the derivation is obtained, and the expression of the stiffness curve is obtained and the stiffness curve can be seen in figure 7 .

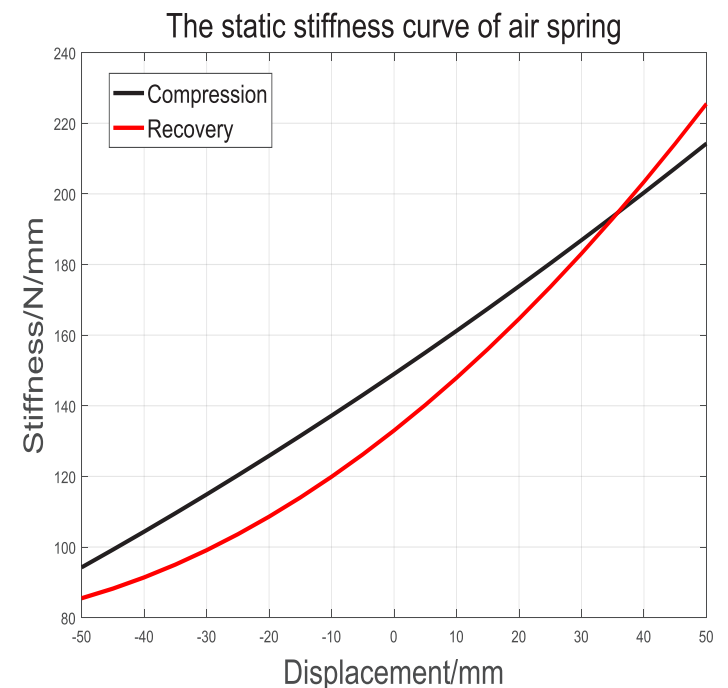

Figure 7.Static stiffness curve of air spring.

\subsection{Contrastive analysis of experiment and simulation data}

The static stiffness curve obtained during the compression process is compared with the static stiffness curve obtained by finite element simulation, as shown in figure 8 .

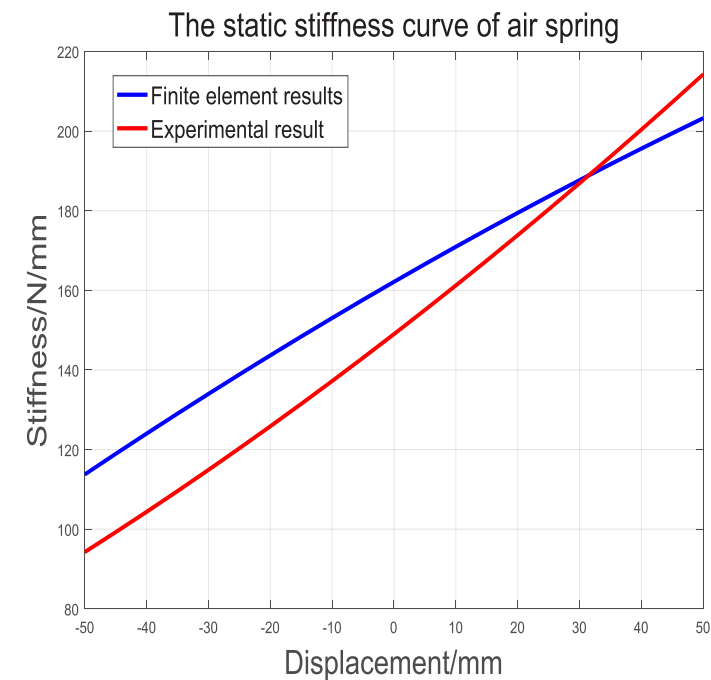

Figure 8.Comparison of static stiffness curve of air spring.

From the analysis above, it can be seen that finite element simulation analysis based on ABAQUS can effectively carry out the analysis of the model air spring. The result of the finite element simulation analysis shows good agreement with the test value obtained by the test, which fully demonstrate the scientific nature of this method.

\section{Conclusion}

In this paper, the finite element model of the membrane air spring is established, and the relationship between the elastic force and the displacement is analyzed with $0.6 \mathrm{MPa}$ as the inflation pressure. Further, the static 
stiffness curve of air spring is calculated and the effect of the installation of the limit sleeve on its stiffness is verified. After that, the air spring bench test is carried out at the same inflated pressure, and the static stiffness curve of the air spring in the process of compression and recovery is fitted. The accuracy and reliability of the finite element simulation method in the analysis of the nonlinear problem of the air spring system are verified by the comparison between the simulation and the experiment. In addition, the use of finite element simulation methods can not only greatly shorten the development cycle of membrane air spring products, reduce the cost of $\mathrm{R} \& \mathrm{D}$ but also avoid the risk of possible problems during the test.

\section{References}

1. B. Liu, J CVGI,07,112-114 (2007)

2. Z. S. Yu, Automobile Theory(2009)

3. C. Y. Yuan, K. K .Zhou, L. Q. Wu, D. F. An, G. L. Wang,CJME,06,262-266(2009)

4. E. Richer, Y. Hurmuzlu, J. Dyn. Syst. Meas. Control,122,416-425(2000)

5. J. L. Zhang, H. Y. Wang, Jou, 06, 115-119(2013)

6. D. Chen, L. I. Fu, Y. H. Huang, EL.MTV (2010)

7. K. Wang, E. F. Chen, Y. Zhang, PVT, 12, 1-6(2016) 